Cahiers de géographie du Québec

\title{
Dans la plaine laurentienne, la glace du sol aurait-elle contribué au façonnement des glissements et autres formes de relief en creux?
}

\section{Louis-Edmond Hamelin}

Volume 15, numéro 36, 1971

URI : https://id.erudit.org/iderudit/020981ar

DOI : https://doi.org/10.7202/020981ar

Aller au sommaire du numéro

\section{Éditeur(s)}

Département de géographie de l'Université Laval

ISSN

0007-9766 (imprimé)

1708-8968 (numérique)

\section{Découvrir la revue}

\section{Citer cet article}

Hamelin, L.-E. (1971). Dans la plaine laurentienne, la glace du sol aurait-elle contribué au façonnement des glissements et autres formes de relief en creux? Cahiers de géographie du Québec, 15(36), 439-465.

https://doi.org/10.7202/020981ar
Résumé de l'article

La formation des glissements de terrain et des rainures sont l'objet de certaines réflexions. Si une cinquantaine de glissements contemporains (depuis 1663) ont fait l'objet d'études, surtout géologiques, environ 700 « coulées » ont été relevées sur photographies aériennes. Les glissements qui correspondent au système " cavité-chaos » sont plus nombreux à la fois sur la rive nord du fleuve et sur les terrasses élevées ; la plupart précèdent la colonisation, donc le déboisement. L'eau constitue la meilleure alliée de l'argile. Les conditions topographiques doivent être également favorables. La gravité joue mais les manifestations tectoniques peuvent favoriser le déclenchement du phénomène. D'autres glissements sont liés à des activités anthropiques. Il n'y a pas de cause unique. Les glissements se produisent non seulement dans les " vallées d'encadrement " mais dans les nombreuses rainures (" coulées ") érodant les terrasses ; la dentritation du tracé des vallons ne doit pas faire conclure à des formes strictement fluviales. L'élargissement des vallées en rapport avec le Didacien comprend trois types. Le modèle des explications usuelles est basé sur des situations présentes mais s'applique-t-il parfaitement aux glissements anciens ? Nous développons l'hypothèse nouvelle de la glace du sol. Des cas, apparemment semblables, ont été étudiés en Alaska, dans le Nord Canadien et en Sibérie. Dans le sol laurentien, en 1957-58, nous avions déjà signalé des indices de paléo ilôts de glace. Ceux-ci peuvent être de deux types : des masses dans les grandes dépressions, un simple réseau de coins sur des terrasses ou sur des formations fluvioglaciaires ; cet englacement a pu s'installer soit pendant un "périglaciaire de situation " au tout début de la déglaciation et de l'émersion, soit pendant des périodes de " périglaciaire climatique » p.e. vers 8000 B.P. ; l'eau était abondante et la végétation mince. Le pergélisol aurait été discontinu. La fusion de cette glace dispersée aurait été liée à des périodes de réchauffement général et à des conditions locales d'exposition à l'air ou à l'eau libre. La fusion aurait favorisé les glissements, les coulées boueuses, les dérangements stratigraphiques et une réduction du relief laissant des buttes témoins. Du côté des rainures, une partie d'entre elles se seraient installées dans des anciennes fentes de froid ou de glace, isolant des quadrilatères, rappelant les baidzherakhs. La glace dans le sol convient très bien aux réticulations végétales qui sont installées sur les hautes terrasses et peut-être aux irrégularités topographiques du canton d'Alfred en Ontario (alass ou thermokarst ?). Plusieurs auteurs ont fait appel à des conditions froides pour rendre compte de micro modelés postglaciaires. Le texte discute aussi de l'objection probable des palynologistes. Bref, en Laurentie, la glace du sol aurait été un agent temporaire et non universel qui, depuis la déglaciation, aurait participé à la genèse de certaines formes en creux.
Ce document est protégé par la loi sur le droit d'auteur. L'utilisation des services d’Érudit (y compris la reproduction) est assujettie à sa politique d'utilisation que vous pouvez consulter en ligne.

https://apropos.erudit.org/fr/usagers/politique-dutilisation/ 


\title{
DANS LA PLAINE LAURENTIENNE, LA GLACE DU SOL AURAIT-ELLE CONTRIBUÉ AU FAÇONNEMENT DES GLISSEMENTS ET AUTRES FORMES DE RELIEF EN CREUX ?
}

\author{
par \\ Louis-Edmond HAMELIN \\ Centre d'Études nordiques, université Laval, Québec
}

Même si elle est posée en des termes limitatifs, cette question aborde de grands sujets; en effet, elle remue des thèmes aussi vastes que les modalités de l'installation de l'Holocène, les variations paléoclimatiques, l'évolution postglaciaire de la vallée du Saint-Laurent, et plus particulièrement le développement d'un système de pente. Faut-il dire très clairement que nous n'avons ni l'information ni la compétence de discuter à fond de tels problèmes. Notre objectif est énormément plus modeste puisqu'il consiste à présenter quelques réflexions concernant certaines irrégularités topographiques des Basses terres du Saint-Laurent. *

Suivant la théorie générale, ces transformations sont de deux types: des reliefs en dépression que I'on avait peut-être trop associés à des modelés de pure érosion (un départ de matière) et des reliefs en hauteur généralement causés par une surcharge accumulative, par exemple, dunes et bourrelets glaciels. Il ne sera question que du premier groupe, c'est-à-dire de l'inscription dans la plaine laurentienne de reliefs en creux et des formes qui leur sont directement associées.

Mais ces cavités comprennent plusieurs catégories : elles ne se limitent pas aux vastes cuvettes de décollement le long des versants des "dépressions d'encadrement ". En 1955, nous avons souligné les "larges rainures qui déchirent les plaines et qui (en certains lieux) ont réduites ces dernières à l'état de morceaux isolés »; c'est le phénomène du vallonnement à large fond. D'autres types de cavités sont les ravins courts et étroits qui rongent les talus de terrasses et qu'on retrouve à la tête des cours d'eau. Il en est de même de certaines microdépressions informes, fermées et à contours

* Sincères remerciements à Arsenault, Samuel, Québec ; Bergeron, Robert, Chicoutimi ; Bernard, Claude, Montréal ; Cailleux, André, Québec; Clibbon, P.B.. Québec : Dion, Louise, Québec ; Dumont, Benoît, Québec ; Flint, R.F., New Haven ; Gagnon, Hugues, Ottawa ; Godbout, G., Ste-Anne-de-la-Pocatière ; Harvey, Louise, Québec ; Johnson, Peter, Ottawa : Laplante, Raymond, Ste-Anne-de-la-Pocatière; Laverdière, Camille, Montréal Parry, J.T., McGill ; Ritchot, Gilles, Québec; Tessier, Yves, Québec; Vincent, J.S. Ottawa : Veyron, Françoise, Québec. 
lobés qui crèvent des surfaces quasi planes. Associées de très près à ces reliefs en creux, les sections intermédiaires non abîmées dont les arêtes évoluent vers un faciès convexe; le paysage d'ensemble devient mamelonné. Enfin, même les tourbières bombées sont installées dans des dépressions relatives. Encore une fois, nous prévenons le lecteur des limites de cette recherche; celle-ci n'a pas pour but de relever, de classifier et d'interpréter chacune des cavités laurentiennes. Par exemple, il ne sera pas question des "marais troués", suivant l'expression d'André Cailleux. Nous ne voulons considérer que les principaux types et globalement.

Comme le titre l'indique, l'originalité de ce travail réside dans le fait d'une nouvelle hypothèse, celle de la glace de congélation dans le sol (non la glace de glaciers qui aurait pu être enterrée). Cette interprétation est dite nouvelle par rapport aux explications usuellement fournies pour rendre compte de la migration subite des glissements de terrain. La notion même de la " glace périglaciaire " est peu connue. "Prior to the introduction by S.W. Muller in 1945 of the well-established Russian concepts of thermokarst few papers in North America even mentioned the pitting of the surface by thaw of buried non-glacial ice ", écrit R.F. Black, avant de conclure: " the history of the thaw (depression) is perhaps the least know and among the most intriguing aspects yet to be deciphered" (Black, 1969, p. 132 et 156 ; voir aussi Dylik, 1964). De notre côté, nous avons souvent mentionné l'action des lentilles de glace notamment dans la réticulation végétale du Québec subarctique (Hamelin 1957, p. 104) et dans les « cours d'eau à berges festonnées" de l'Abitibi I. En outre, dans notre rapport sur les îles-de-la-Madeleine en 1958, nous utilisions: "blocs gélivés», "vallon périglaciaire", "ruissellement nival en présence de sol gelé ». D'une façon plus générale, et pour certaines régions du Canada méridional, nous avons fait I'hypothèse des "lentilles de glace dans le sol "contemporaines de la déglaciation et d'«ilots permanents de gélisol » pendant une période

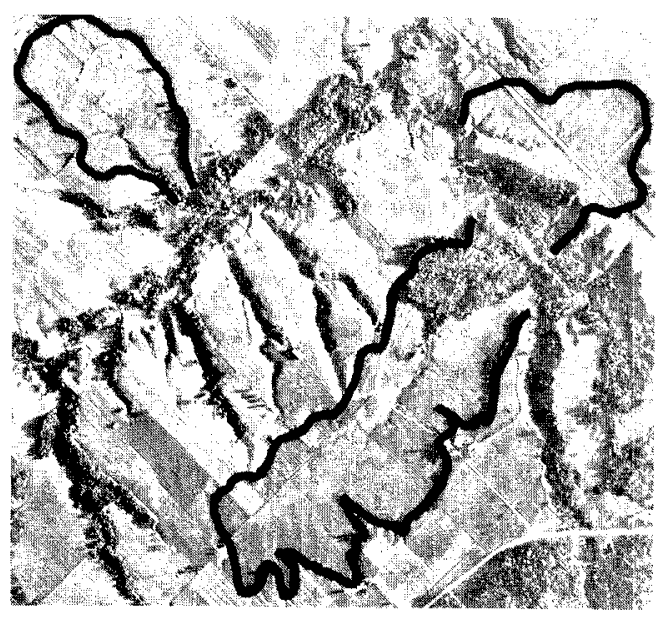

Photo Q-64541-139, Ministère des Tarres et Forêts, Québec

Photo 1 Rainures et cavités. Région de Sainte-Marcelline de Kildare. Montcalm, Québec. En trait accentué, les plus grandes cavités.

1 HAMELIN, L.-E., « Les cours d'eau à berges festonnées", The $\mathrm{Ca}^{-}$ nadian Geographer - Le géographe canadien, no 12,1958, p. 20-24. 
du postglaciaire (Hamelin, 1961, p. 42 du tiré-à-part). Des voyages dans le monde nordique et surtout un en Sibérie orientale en 1969 nous ont poussé à mairitenir notre hypothèse d'une paléoglace dans le sol pour une meilleure compréhension d'un certain nombre de cavités laurentiennes. Aussi, le 9 mai 1970 , à l'excursion de la Société des Professeurs de Géographie du Québec organisée par J.M. Lancery de l'Université du Québec à Trois-Rivières, dans le bassin du Gentilly, nous avons ajouté aux commentaires sur les vallons, l'hypothèse de la fusion d'anciennes masses de glace. Au même

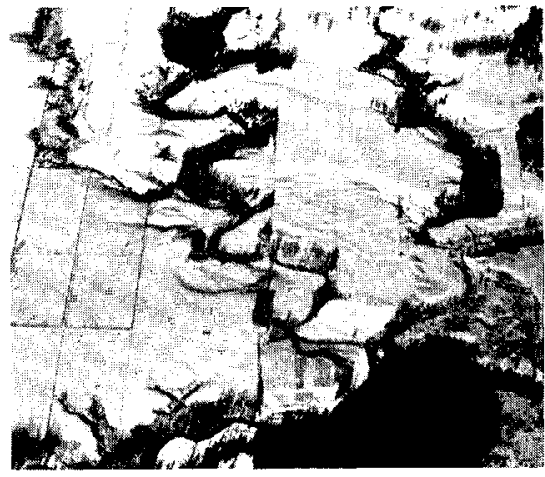

Photo Louis-Edmond HAMELIN (1970)

Photo 2 Glissements et rainures au détriment de la plaine. Région de Lachute, Québec. moment, semblable explication était envisagée pour les grandes cavités qui sont inscrites dans la terrasse principale de la Grande rivière de la Baleine ${ }^{2}$. Bref, il s'agit d'une hypothèse périglaciaire qui, dans le Québec, effraie moins qu'avant.

Deux parties dans ce texte. La première sera consacrée à une courte présentation des phénomènes et des explications usuelles concernant le type de cavités laurentiennes le plus répandu, celui des glissements de terrain. En second lieu, I'hypothèse de la glace dans le sol, comme agent participant au façonnement des reliefs en creux, sera présentée et discutée.

\section{1- BREVE REVUE DES CONNAISSANCES SUR CERTAINES CAVITÉS DE LA PLAINE LAURENTIENNE}

L'objet de notre recherche n'étant pas une étude complète des glissements, le rappel des opinions courantes ne peut être autrement que rapide. Elle sera quand même utile pour préparer le lecteur à l'hypothèse nouvelle de la deuxième partie.

Nous entendons par glissement une descente de matériaux, plus rapide que le creeping et la gélifluxion, mais une migration non permanente. C'est un phénornène de masse comme l'avalanche, l'écroulement, l'effrondement et l'éboulement; le déplacement ne se fait pas bloc par bloc comme dans le cas de l'éboulis; généralement, le glissement intéresse une nappe plus profonde que la solifluxion. Dans le paysage, le transfert de la matière minérale et de sa couverture végétale (et nivale) laisse une cicatrice en amont et construit un gonflement, en aval d'un couloir intermédiaire parfois

2 HAIMELIN, L.-E., et CAILLEUX, A., "Poste-de-la-Baleine (Nouveau-Québec) : Exemple de géomorphologie complexe », Revue de géomorphologie dynamique, Sstrasbourg, 1969-70, no 3, p. 144. Verbatim, J.P. Portman et Marc Boyé. 
court. Dans les glissements dits "humides», un lubrifiant, l'eau, excite la viscosité du matériel; l'argile devient alors "savonneuse», suivant une expression québécoise. En soi, ce phénomène ne préjuge d'aucun système morphoclimatique particulier puisqu'on le rencontre aussi bien au Rif (Maroc) que dans les régions arctiques, surtout dans celles où le plafond du pergélisol est bas. Quand des facteurs "froids" comme des lentilles de glace de sol, la " table » du pergélisol, le cycle gélival ou l'infiltration de l'eau de fonte concourent à la création des glissements, ces derniers peuvent être qualifiés de périglaciaires. Dans l'évolution du relief, c'est très rarement qu'un processus joue isolément : "les formes pures sont rares ". Avant, même pendant et après les glissements, d'autres agents comme les différents modes de ruissellement et la soli (géli) fluxion continuent d'exercer leur action. Ainsi, quand ancienne se trouve la descente brutale des matériaux, la contribution d'un glissement ancien au façonnement du relief actuel est davantage partielle.

\section{Exemples de glissement}

Même si, de toute évidence, la majorité des glissements laurentiens ne sont pas actuels, la littérature scientifique ne concerne pratiquement que les formes historiques; cette limitation tient probablement au fait que jusqu'à récemment, I'on n'étudiait pas le Postglaciaire dans sa totalité. Les glissements historiques, nous les faisons commencer au XVIle siècle (Pouliot, 1963).

\section{Glissements postérieurs à 1662}

L'intérêt de ces manifestations brutales réside dans une littérature relativement abondante — même si les sources premières sont minces — et dans le fait de la provocation catastrophique des glissements. Les Relations des Jésuites (volume XLVIII) parlent d'un "tremble-terre universel en Canada. En 1663, la guerre sembloit estre mesme entre les montagnes dont les unes se déracinoient laissant de grands abysmes ... une forest entière s'est glissée dans le fleuve ». À son tour, l'excellent écrivain que fut Marie de l'Incarnation donne l'exemple " de la rivière Batiscan où se sont créés de profonds abîmes", en février 1663. Les montagnes se détachèrent de la terre ferme et formèrent dans le fleuve de nouvelles îles. Évidemment, une société théocratique comme celle du Québec d'alors n'a pu s'empêcher de tirer les leçons religieuses des événements: les Annales de l'Hôtel-Dieu ont constaté que les faits "remuèrent les consciences des pécheurs. Jamais, il ne se fit de confessions plus sincères ". Quoi qu'il en soit, ces glissements de terrain ont été directement reliés aux tremblements de terre.

Le relevé de tous les décollements rapportés dans la littérature constituerait une recherche historique en soi. II est étonnant que les index de revue en parlent très peu ; c'est le cas de celui de la Literary and Historical Society of Quebec et surtout de celui de la Société de Géographie de Québec, ce dernier établi par Ch. Morissonneau en 1969. De toute façon, d'autres sources mentionnent des glissements en 1791 et en 1870 . 
L'étude scientifique de ce phénomène commence avec les travaux des géologues dont Sir Logan qui décrit la coulée du Maskinongé en 1840. Quinze glissements sont rapportés dans Soils in Canada (Royal Society of Canada, 1961). L'on peut dire que jusqu'à celui de Saint-Jean-Vianney, le 4 mai 1971, une cinquantaine de cas contemporains ou récents ont été signalés et parfois bien étudiés (Morin, 1947 ; Laverdière, 1956 ; Béland, 1956).

Petit à petit, l'on a dépassé l'aspect événementiel pour s'intéresser à un plus grand nombre de situations, d'abord celles du passé immédiat. En 1950, I'University of Illinois a repris l'étude du glissement de SaintThuribe le long de la rivière Blanche, en particulier celui de 1898; mais elle indiquait dans les environs immédiats deux "Old Flow». En outre, de nombreux géologues, pédologues et géographes ont signalé à l'occasion des glissements anciens et récents. Pour ne mentionner que quelques auteurs, Gilles Ritchot en 1964 dans la Plaine de Joliette; la même année, et en 1969, Peter B. Clibbon en bordure des Laurentides; Jean-Claude Dionne en 1970 à l'ouest de Chicoutimi et Germain Tremblay en 1971 dans la Plaine du Lac Saint-Jean ${ }^{3}$. L'importance de ces phénomènes a été reconnue dans I'Annuaire du Québec de 1971 où l'on écrit à la page 49 : "les éboulements et les glissements de terrain sont les modes les plus importants dans l'évolution des versants du Québec habité ».

\section{Des glissements plus anciens}

L'idée de déplacements anciens s'est affirmée. À Saint-Jean-Vianney, $P$. Lasalle et J.Y. Chagnon ont étudié un paléoglissement datant de 1460 environ. Par ailleurs, entre Ottawa et le Creek, un semblable déplacement de terrain meuble a eu lieu vers l'an 1000, d'après la Geological Survey of Canada (Canada, 1966). L'ancienneté de certaines formes est souvent affaire d'observations. À Saint-Joseph-de-la-Rive, le Saint-Laurent actuel entaille une falaise à même un chaos d'éboulement-glissement subactuel, formation qui incorpore des troncs d'arbre fossiles. Le long de la Rivièredu-Loup (Mauricie), un glacis de glissement est découpé en terrasses fluviales. En plusieurs endroits, dont à Saint-Barnabé (Yamachiche), des formes jeunes, de reprise, s'inscrivent dans des formes anciennes, encaissantes et que l'on aurait cru figées. Gilles Ritchot a constaté qu'au long du GrandCoteau (région de Montréal), la mer de Champlain a vraisemblablement érodé des loupes de glissement. De l'avis des premiers défricheurs, des "cavées d'éboulis" existaient déjà avant le déboisement anthropique. En fait, l'on constate des glissements anciens le long de la plupart des cours d'eau affluents du Saint-Laurent; pour nous en tenir au versant nord où les cas semblent plus nombreux, mentionnons la rivière des Outaouais, la Petite-Nation, le Lièvre, la rivière Rouge, I'Assomption, le Ouareau, la Bayonne, la rivière Blanche (affluent du Maskinongé), le Maskinongé, les

3 Verbatim. S. Arsenault pour les hautes terrasses de la Sainte-Anne-de-Beaupré. 
Yamachiche, le Saint-Maurice, la rivière Cachée, le Batiscan et ses affluents, le Portneuf, la rivière Blanche et la Sainte-Anne-de-la-Pérade. Au Lac-SaintJean, la Couchepaganiche et les environs de la Baie des $\mathrm{Ha}$ !Ha! offrent des exemples caractéristiques d'évidement en terrain meuble. Le nombre total des glissements actuels et anciens n'est pas encore établi; des relevés sur des photos aériennes ont parlé de sept cents "coulées d'argile". De ce nombre, environ 3\% seulement ont été étudiées. L'échelonnement dans le temps de certains glissements (ceux de 1663 ont duré plusieurs mois), la reprise possible des activités au même site et la destruction graduelle des formes rendent très difficile le dénombrement des glissements à l'unité. Quoi qu'il en soit de leur nombre total, les phénomènes de mémoire d'homme ne représentent qu'une très faible partie de l'ensemble.

D'après nous, les glissements n'ont pas commencé avec le déboisement. II s'agit plutôt d'un phénomène quasi permanent depuis la libération du territoire par le retrait de la mer de Champlain, et peut-être par la déglaciation. Certes, faut-il envisager que la fréquence des glissements a varié au cours des derniers dix mille ans, variation causée par une combinaison d'éléments qui ne pouvaient toujours être également favorables. Des glissements se sont produits avant l'installation naturelle de la végétation, au cours de la période végétale (qui a elle-même varié d'intensité) et depuis le défrichement. Même, il ne serait pas interdit d'imaginer que la phase de pré-déboisement ait été la plus favorable des trois.

Par leur nombre et par la durée de la période géologique au cours de laquelle de tels événements se sont produits, les glissements de terrain ont constitué un processus majeur dans l'évolution des micro-reliefs laurentiens.

\section{Les reliefs en creux}

Cette section sera consacrée à la présentation des formes de relief, dans la pure tradition de la géomorphologie. Tel qu'annoncé en introduction, ce ne sont pas toutes les irrégularités topographiques en moindre qui seront discutées; nous intéressent particulièrement celles qui peuvent être liées à une occupation ancienne et différentielle de la glace dans le sol. Les deux principaux types de forme à considérer sont les glissements et les rainures dendritiques.

\section{Le système cavité-chaos}

Sur le plan de la dynamique topographique, les glissements forment un système qu'on pourrait qualifier de "cavité-chaos》, caractérisant ainsi et l'entonnoir d'évidement inscrit dans la partie supérieure du versant et le chaos d'accumulation sis en contrebas de lui. Le sens fondamental du mot glissement rend l'idée d'action, non celle d'une forme de terrain d'où, ici, l'expression topographique cavité-chaos. Ces deux mots semblent préférables au groupe "cratère-éboulis"si l'on veut éviter des confusions de processus. 
Nous n'aimons pas trop « coulées d'argile » car ce qui glisse n'est pas toujours de l'argile, granulométriquement, et, au Canada français, coulée signifie soit une vallée, c'est-à-dire une dépression, soit au contraire une surcharge d'accumulation. Pour la terminologie des glissements, Henri Baulig fournit la principale référence ${ }^{4}$.

Relativement au talweg laurentien actuel, la situation des nichesloupes retient notre attention. Leur répartition ne semble pas universelle; plusieurs auteurs ont constaté qu'elles sont plus nombreuses sur la rive nord que sur la rive sud du fleuve. En deuxième lieu, il appert également que leur répartition en altitude n'est pas non plus uniforme; d'après la carte des sols du comté de Joliette, la majorité des cavités de glissements s'inscrivent au-dessous de $122 \mathrm{~m}$ (400 pieds) mais au-dessus de $23 \mathrm{~m}$ (75 pieds), d'après Pageau en 1961. De son côté, Nelson Gadd a mentionné en 1957 que les glissements sont plus nombreux sur les niveaux élevés. R. Blanchard écrira : " les plus hautes terrasses, dans la région de Shawinigan, sont en lambeaux et dressent des formes géométriques fondues, des trapèzes mous $"{ }^{5}$. En troisième lieu, l'amplitude des cavités est également différentielle : les méga-glissements perchés sont plus nombreux que ceux qui sont bas logés. Cette triple inégale distribution est-elle liée à des variations sédimentologiques, à des moments climatiquement différents du Postglaciaire, à la situation topographique locale ? Quoi qu'il en soit, elle suggère l'intervention de processus autres que ceux auxquels une conception de pays tempérés avait fait spontanément penser.

Les dimensions des glissements sont fort variables; l'événement de Saint-Jean-Vianney au $15 \mathrm{e}$ siècle a intéressé une étendue de $20,4 \mathrm{~km}^{2}$ ( 8 milles carrés) et une épaisseur de $9 \mathrm{~m}$ (30 pieds) d'après Lasalle et Chagnon, en 1968. La profondeur de la dépression laissée à la suite du glissement de Saint-Thuribe en 1898 était également de $9 \mathrm{~m}$. L'exemple situé entre Ottawa et le Creek montre une forme niche-loupe longue de $853 \mathrm{~m}$ (2800 pieds), large de $518 \mathrm{~m}$ (1700 pieds) pour une profondeur d'environ $7,6 \mathrm{~m}$ ( 25 pieds). Les pentes de l'entonnoir sont fortes, atteignant même la position subverticale au droit de la fissure de décollement; la pente de provocation est elle-même variable et, en 1958, nous écrivions que dans Charlevoix de nombreux décollements-glissements se font sur des pentes de $35^{\circ}$ environ. À Saint-Barnabé, les arêtes actuellement menacées par des microglissements de reprise sont tendues à $35^{\circ}$. La partie "loupe » ou chaos des glissements comprend des micro-reliefs bien variables au nombre desquels se retrouvent pêle-mêle des paquets basculés en sens contraire de la pente, des marches d'escalier, des bourrelets pseudo-concentriques, des chaos en coupole, des fines ondulations et des frémissements;

4 BAULIG, Henri, Vocabulaire franco-anglo-allemand de géomorphologie, Pariș, Les Belles Lettres, 1956 (première édition), 230 pages.

5 BLANCHARD, Raoul, Le centre du Canada trançais, Montréal, Beauchemin, 1948, p. 407. 
tranchent la masse accumulée, des sillons transversaux, longitudinaux ou radiaux. Le glissement le plus massif aurait déplacé 22574700 mètres carrés (27000 000 de verges carrées).

$\mathrm{Vu}$ en plan, le système cavité-chaos tend à présenter principalement une forme légèrement allongée comportant, comme le chiffre 8 , un certain resserrement central ; les extrémités amont et aval étant courbes, l'on peut parler de concavité de la niche et de convexité du chaos. Dans le détail, le rebord de la cavité est souvent ourlé de festons. Sur le plan de la durabilité, la cavité est moins vulnérable que le chaos, celui-ci tendant à s'aplanir plus rapidement que l'entonnoir à se combler. La dépression d'en haut, plus que le gonflement d'en bas, sert donc d'indice topographique aux glissements anciens. Certains fonds ont des pentes aussi faibles que $6^{\circ}$, voir même $2^{\circ}$, comme au Ruisseau-Plat, à Saint-Didace - Saint-Édouard. Les glissements ne se sont pas tous produits à l'intérieur des grandes « dépressions d'encadrement ", ces vastes auges qui comme la vallée du Saguenay occupent souvent des sites préglaciaires et qui ont reçu d'abondants dépôts-meubles au finiglaciaire et au Champlain ; l'on en trouve aussi dans des vallées plus récentes.

\section{Les rainures de tracé dendritique}

Ces rainures - ou vallées ou ravins ou vallons ou " coulées " suivant une vieille expression française utilisée dans les Relations des Jésuites ${ }^{6}$ ont été moins étudiées que les glissements. Cependant, Marcel Bélanger avait consacré d'excellentes pages aux rainures de $15 \mathrm{~m}$ à $61 \mathrm{~m}$ (50 à 200 pieds) de profondeur inscrites dans le delta du Saint-Maurice ${ }^{7}$. II s'agit d'une forme allongée alors que le système cavité-chaos compose un ensemble plus massif. Le chevelu du réseau est très caractéristique, comme le montrent si nettement les Yamachiche. Frappe la largeur de ces vallées. Le Creek près d'Ottawa coule dans une dépression de 304 m (1000 pieds) de large. En aval de Saint-Léon-de-Maskinongé, Gérard Godbout a cartographié l'argile Rideau sur une largeur de plus de 914 m (3 000 pieds), argile qui correspond assez bien à la dépression. L'on ne peut donc éviter d'être frappé par la disproportion entre la largeur des vallons et la puissance de la fluviation actuelle et même de la fluviation ancienne. En outre, ces rainures montrent un fond très plat que nous avons dénommé Didacien (Hamelin et Dumont, 1961). La régularité du plancher de ces dépressions allongées peut être liée à l'accumulation qui se fait en amont des embâcles glacielles ou des barrages de glissement; d'autres $y$ ont vu une forme anthropique.

Au-delà de ces observations et explications courantes, il faudrait songer que fonds, élargissement et versants sont liés. Nous sommes ainsi conduits à rapprocher la genèse des glissements et celle des ravins, et beaucoup plus qu'on ne l'a fait dans le passé. C'est une multitude de glissements latéraux

6 Archives du Français canadien. Département de linguistique de I'Université Laval.

7 BELANGER, M., "Trois cartes originales sur le relief de la région du Bas SaintMaurice", The Canadian Geographer - Le géographe canadien, no 2, 1952, p. 35-42. 
migratoires - la plupart mineurs - qui ont démesurément élargi ces rainures d'apparence purement fluviale. La dendritation du tracé de la vallée et la méandrisation du lit ne doivent pas faire conclure à un simple ruissellement. Vues longitudinalement, les cavités d'extension latérale ont d'abord été locales et la vallée n'était large qu'à ces endroits; quand les évidements se sont exercés tout au long de la paroi, c'est toute la dépression qui prenait du volume et qui devenait ainsi calibrée. Verticalement, I'on constate souvent que l'élargissement n'a pas intéressé tout le versant; en bien des sections, le départ de matière a été moins important dans la partie inférieure que dans la partie supérieure; nous pourrions appeler "vallée de l'Holocène » ces élargissements de plusieurs centaines de mètres correspondant pour une large part à des cavités de glissement. La contribution des glissements au façonnement des rainures pourrait être présentée en trois types schématiques: un stade jeune comprenant une cavité localisée le long d'une vallée étroite, autrement en $V$. Un stade intermédiaire montrant le recul généralisé de la paroi et laissant une large vallée à fond non nettement régularisé. Un stade évolué ayant des formes convexo-concaves de versant plutôt figé et un fond Didacien au travers duquel un petit filet d'eau s'inscrit timidement. Toutes les rainures n'évoluent pas suivant ce schéma; elles n'en sont pas toutes au troisième stade et même lorsqu'elles y sont arrivées, de petites descentes brusques de matière peuvent s'exercer aux lieux mal stabilisés. Il est important de retenir que de nombreux glissements se sont produits à l'intérieur des rainures.

Ces deux types majeurs de dépression - les cavités-chaos inscrits dans les vastes dépressions qui encadrent les cours d'eau actuels et les rainures entaillant les terrasses ou le fond de petits bassins - laissent un paysage bien caractéristique. La partie supérieure des versants de très nombreuses vallées — notamment dans la région de la Baie des $\mathrm{Ha}$ ! $\mathrm{Ha}$ ! et de Shawinigan — suggère que le relief originel fut largement démoli ; les surfaces initiales ont grandement été réduites; les étendues résiduelles occupent moins d'espace que les étendues défoncées. Les buttes-témoins sont de toutes formes : îlots très isolés et îlots comportant d'étroits pédoncules; quant aux masses plus considérables, elles sont tranchées de dépressions orientées de façon souvent insolite par rapport au réseau fluvial ; dans la Plaine de Joliette, des entonnoirs de glissement se sont eux-mêmes menacés de capture. Les têtes de vallon montrent une curieuse tendance au dédoublement en " corne de boeuf»; très curieusement, ces "coulées" isolent des quadrilatères; tout cela n'est pas sans rappeler le tracé des réseaux hydrographiques sur fente de gel, ou de glace ou de remplissage minéral. Même impression lorsqu'on regarde diverses sections des talus des terrasses où sont inscrites des rainures peu profondes et régulièrement espacées, par exemple dans la région de Lachute ; l'entre-rainure fait penser aux baidzherakhs - coupoles entre des fentes de glace - sur les versants de la Léna en Sibérie. De toute façon, par le volume du matériel enlevé et par un agencement souvent anarchique du relief résiduel, il est difficile de penser à un modelé fait par les seules eaux courantes en climat tempéré. 


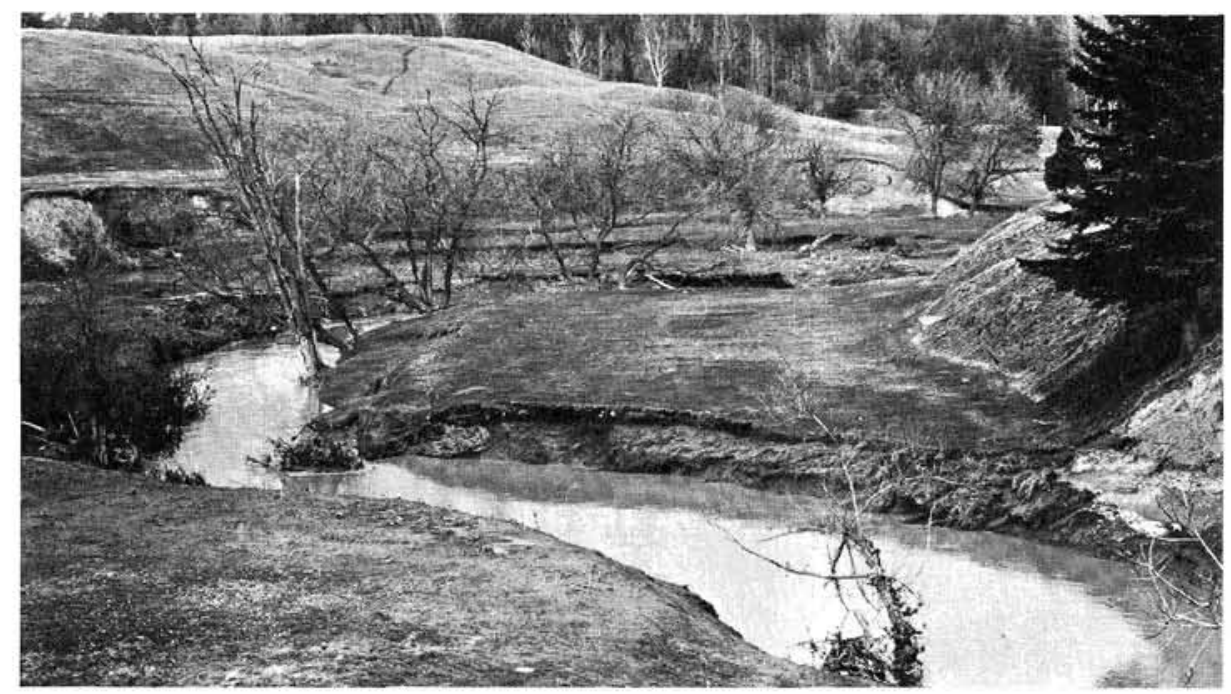

Photo Louis-Edmond HAMELIN (1969)

Photo 3 Largeur disproportionnée du vallon. Glissements pelliculaires de reprise. Didacien (basse terrasse). Saint-Sévère, Québec.

\section{Des dépressions de thermokarst?}

Il y a d'ailleurs des genres de cavité plus énigmatiques : ce sont celles de la partie basse de la plaine des Outaouais dans le canton d'Alfred en Ontario et de la Presqu'île de Plaisance dans le Québec. La topographie est caractérisée par un moutonnement anarchique, à dépressions serrées et peu profondes. "Near the Ottawa River, Alfred Township is somewhat hilly », raconte I'histoire locale ${ }^{8}$. Chapman et Putnam ont classifié la région "clay plain ». Un rapport géologique récent précise qu'il s'agit d'une "clay, stonefree, grey or dark grey, layered, ondulating or level, with imperfect or poor drainage " 9 . L'on pourrait penser qu'à la surface des dépôts réapparaissent des anciennes dolines mais le sous-sol n'est pas partout calcaire. La microtopographie suggère plutôt une moraine d'ablation qui aurait été associée à une déglaciation sur place; mais les cailloux sont rares et les dépôts sont ordonnés suivant une certaine stratigraphie. L'on ne peut non plus retenir I'hypothèse du vêlage d'icebergs, pas davantage celle de l'action fluvioglacielle, non plus celle du simple découpage en terrasse. La forme lobée de mini-dépressions fermées classifiées comme "Bearbrook " sur la carte pédologique, forme lobée qui apparaît davantage sur la carte Hawkesbury au 50 millième que sur le terrain, suggère des dépressions de fonte de culot

8 THOMAS, C., History of the countries of ... Prescott, Montreal, Lowell 1896, p. 630 .

9 Soil Map of Prescott County. Ontario, Report no 33, Department of Agriculture, Ottawa, 1962. 


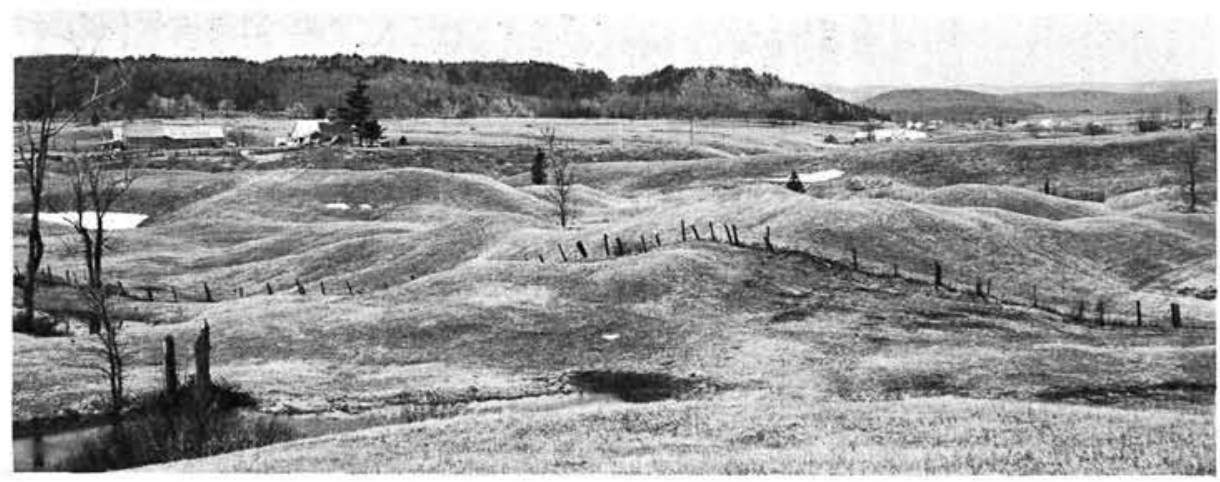

Photo Peter B. CLIBBON (1963)

Photo 4 Moutonnement généralisé du relief. Rivière-du-Loup, Bout du Monde, Mauricie, Québec.

de glace dans le sol. S'agit-il d'une glace de glacier sans moraines qui aurait été fossilisée par des dépôts champlainiens ou d'une simple congélation d'eau dans le sol ? Les deux sont possibles.

\section{Les « tourbières 》 figurées}

Les formations organiques des pays froids présentent en surface des figurations originales; l'alternance des mares et des lanières de végétation se fait suivant un dessin géométrique dont nous avons présenté les principaux types dans le volume 1 de La Revue de Géographie de Montréal, en 1971 ; il s'agit de tracé rectiligne ou tigré, concentrique, vermiculé, réticulé et ponctuel. II existe des réticulations végétales près du lac Saint-Joseph, Québec (Hamelin, 1957) ; le Canada laurentien, au Québec et en Ontario, en comporte beaucoup d'autres exemples. P.B. Clibbon nous en a signalé deux près de Beaumont, Québec. Or, il est probable que ces figurations aient été associées à des manifestations du froid. D'une façon plus précise, nombreuses sont les "îles de végétation arbustives» dans une mer de sphaignes; I'on peut même en voir au sud-est de Québec, le long de la route $\mathrm{n}^{\circ} 20$ dans des tourbières interdunaires. Près de Shawinigan, la figuration des Plés est remarquable et ceux (J. Risi) qui en ont fait l'étude ont constaté que les îlots correspondaient à une formation plus minérale alors que les environs non boisés étaient davantage ombrotrophiques; ailleurs, I'on constate la même chose quand il s'agit de sites de palses et de pingos. Le matériel des tourbières a été particulièrement sensible aux actions du froid et nous croyons que des lentilles de glace dans le sol s'y étaient installées et $\mathrm{y}$ ont vécu pendant une partie de l'Holocène.

Ainsi, l'examen de quelques formes en creux nous conduit à penser que la théorie géomorphologique des pays strictement tempérés ne rend pas compte de tous les types de micro-modelés laurentiens. Mais qu'en est-il de l'aspect génétique comme tel. 


\section{Explication usuelle des glissements}

Les ingénieurs et les géologues s'engagent de plus en plus dans des explications hautement scientifiques lorsqu'ils ont à rendre compte des migrations brutales des masses minérales; on sait que de tels déplacements créent l'un des types les plus originaux des reliefs en creux de la Laurentie. Voici un résumé des opinions concernant la genèse des glissements.

La façon d'agencer les commentaires explicatifs varie d'un auteur à l'autre. À la Commission des versants de l'Union géographique internationale, le professeur Paul Macar distingue des éléments actifs, des éléments passifs et des éléments indirects (par exemple, le climat). D'autres chercheurs utilisent un ordre plus analytique en considérant respectivement les conditions sédimentologiques, topographiques, hydrologiques, périglaciaires et humaines. Nous grouperons nos remarques sous deux rubriques.

\section{Les aptitudes à la mobilité}

La migration des matières minérales et de leur chapeau végétal tient d'abord à la nature des matériaux, argile ou limon. D'après une récente mise au point de la faculté des Sciences de l'université Laval, les dépôts de la mer de Champlain sont constitués de minéraux dont 55 à $80 \%$ sont de dimension inférieure à $0,002 \mathrm{~mm}$, soit une taille trop petite pour être identifiée au microscope courant. Les argiles qui ont vécu l'aventure des glissements possèdent une composition minérale semblable, soit $40 \%$ de quartz, $20 \%$ de feldspath sodique, $30 \%$ de minéraux en feuillet et $10 \%$ d'autres minéraux; ces matériaux suggèrent le Bouclier canadien comme principal fournisseur.

En soi, cette composition minérale est stable mais la présence d'un autre élément, l'eau, la menace de déstructuration. Pendant la sédimentation champlainienne, les argiles se sont empilées en flocons. Le floconnement a été grandement favorisé par la présence de divers sels marins qui ont constitué des liens entre les particules minérales. Les solutions salines apparaîtront donc comme des éléments de stabilité. Vu autrement, on pourrait dire que les espaces entre les particules étaient remplis d'eau et que le volume hydrique de la masse dépassait le volume minéral. Pour les dépôts de la région d'Ottawa, W.J. Eden (Canada, 1958) écrivait en 1957: "Generally, the natural water contain of the clay is higher than the liquid limit ". S'il se produit une désalinisation et une immigration d'eau non salée. les liens de la sédimentation originelle se relâchent, la consolidation intergranulaire se défait, l'atomisation réapparaît, les particules en viennent à flotter librement dans une matrice liquide: dans un nouveau bain, l'argile a perdu sa résistance antérieure; elle est prête à s'écouler comme une pâte visqueuse. Les scientistes ont établi pour ce minéral fin des limites dites de liquidité, de plasticité, de sensibilité ou de résistance au cisaillement. La thyxotropie de l'argile se dit de ces aptitudes à la mobilité. Dans le mélange eau-argile, écrit Sergéev (1971), l'eau est « solidement, faiblement ou non liée à l'argile ». 
L'addition d'eau vient d'abord des précipitations et de la nappe phréatique. Si, en théorie, l'imperméabilité de l'argile tend à refuser la pénétration hydrique, toute fissure, tout plan de tassement et de décrochement, tout ancien chenal fossilisé offre des occasions favorables à une surhydratation. L'ingénieur J.Y. Chagnon a démontré l'influence des anciennes vallées, comblées par la suite, dans la circulation actuelle de l'eau interne: il semble bien qu'un écoulement continue à se faire en profondeur entre les lacs Kénogami et Saint-Jean et que cet apport favorise des glissements en bordure de cette dernière dépression. En 1955, un semblable phénomène se serait produit, à Nicolet où le glissement aurait été influencé par une circulation d'eau souterraine et subactuelle. Autres cas : on constate que des glissements sont localisés à l'aval des dépressions organiques, comme si cette dernière alimentait une certaine décharge faite en profondeur et en direction du futur entonnoir du glissement. Ces observations nous invitent à ne pas lier exclusivement les glissements aux sites d'écoulement superficiel. Inversement, sur un versant exposé au soleil, l'évaporation enlève une bonne partie de l'eau qui, en s'infiltrant, aurait pu servir de lubrifiant. Le phénomène de déshydratation a toutefois l'inconvénient d'inscrire dans l'argile des fentes de dessication qui s'offriront comme un réseau d'infiltration épigénétique lors des prochaines pluies. L'on peut interpréter de bien des façons la fréquence mensuelle des glissements; l'une de celle-là serait de constater !'influence de l'eau apparemment disponible à la date de la catastrophe ; les cas étudiés montrent que les phénomènes se produisent pour $43 \%$ dans les mois d'avril et de mai. Dans l'affaire des glissements, l'eau constitue donc le meilleur allié de l'argile. Bien que dans le Québec, les loess semblent peu répandus, ils devraient être eux aussi très sensibles à la fluxion.

Vue de bas en haut, la solidité du profil minéral n'est pas uniforme mais, généralement, elle atteint son minimum vers quelques dizaines de pieds de la surface; l'épaisseur de la tranche victime de glissement semble être conforme à ces dispositions structurales.

Dans le Québec laurentien, une situation stratigraphique très répandue favorise le délayage d'une tranche d'argile. Il s'agit de la superposition de matériel sableux et perméable sur une nappe de matériaux plus fins ; l'argile sousjacente déstructurée par l'eau glissera facilement sous le poids de la surcharge sableuse.

Les conditions topographiques doivent être également favorables. Pour qu'il se fasse un déplacement de masse, il faut un terrain d'accueil ; en d'autres termes, s'il n'y a pas d'endroits où les matériaux puissent atterrir. le déplacement de grande charge est quasi impossible; des conditions idéales résident dans un relief en creux sis en contrebas d'un matériau perché. Dans les "dépressions d'encadrement" et les "vallées de l'Holocène ", le commandement du relief entre les débris des hautes terrasses champlainiennes et le talweg du collecteur principal constitue actuellement une amplitude topographique favorable. II n'en était pas ainsi au début de la période du Post-Champlain. De même dans les petits bassins fermés des 
Laurentides; c'est avec raison qu'en 1963, Gilles Ritchot observait dans sa thèse que les seuils rocheux avaient gêné le développement des badlands, partant des glissements locaux.

Les conditions topographiques ont également leur importance dans les parties sommitales des zones menacées de glissement. Si l'argile est mince et si elle est mal accrochée aux terrains sous-jacents ou si ceux-ci forment des surfaces convexes et lisses, on peut prévoir une immigration massive des argiles et même un nettoyage complet d'elles; les crans arrondis et perchés de Saint-André-Avellin dans les Laurentides au nord de la rivière des Outaouais ne sont guère en mesure de retenir leurs chapeaux d'argile; à leurs pieds, le relief est en creux.

\section{Causes et occasions d'instabilité}

Facteurs et aptitudes se conjuguent pour causer des glissements. La tradition d'une interprétation catastrophique des événements fait d'abord penser aux forces tectoniques. Le fameux exemple de 1663 a été rattaché à des tremblements de terre généralisés. Le Père 1. Lalemand, Jésuite, y voyait une intervention du Ciel car la catastrophe avait de "bons effets sur l'estat du christianisme ". II ne faut pas minimiser le rôle des tremblements de terre dans le décollement de matériaux mal consolidés de la Plaine du SaintLaurent ; I'on sait qu'à ce point de vue, cette région est l'une des plus sensibles du Canada ; I'on y enregistre par année des centaines de mini-secousses et certaines ont atteint une intensité de 7 ; ce fut apparemment le cas en 1663. D'après J.H. Hodgson 10, d'autres importants tremblements de terre ont eu lieu en 1638, 1732, 1791, 1831, 1860, 1870, et 1925. Tant que les spécialistes n'auront pas fait une étude systématique d'un nombre beaucoup plus grand de glissements et de tremblements de terre, l'on ne pourra évaluer la relation précise de cause à effets entre ces deux événements. Pour 1663, la chose semble claire. En théorie, cette relation ne doit pas étonner. La vallée du Saint-Laurent est un couloir où les faiblesses tectoniques se sont souvent manifestées. II est en position de transition, d' " avantpays", entre "un pays" (le Bouclier) et la chaîne plissée des Appalaches, suivant un langage qu'on peut emprunter aux tectoniciens alpins. En 1966 , Kumarapeli a développé I'hypothèse que " the Saint Lawrence Valley system is a North American equivalent of the East African rift valley system ". Les Montérégiennes témoignent elles aussi de manifestations tectoniques. En outre, des géologues ont localisé des failles importantes et des géomorphologues ont déduit de certaines formes résiduelles du relief la présence d'autres failles et de flexures, notamment en bordure des Laurentides ; ces accidents anciens ont pu rejouer lors du redressement isostatique postglaciaire. La foudre n'est même pas exclue. Les forces tectoniques originelles et de reprise par les ébranlements qu'elles causent et par les

10 HODGSON, J.H., «Canadian Earthquakes», Canadian Geographical Journal, vol. LXXI, no 1, 1965, p. 30-39. 
réajustements des forces de pression qu'elles réalisent ont sans doute été un facteur dans la descente brutale des matières visqueuses.

Glissements et rainures sont liés, avons-nous dit. Si les premiers ont contribué à élargir les secondes, celles-ci ont souvent provoqué ceux-là. Au début de l'Holocène, pour que des glissements subaériens commencent à se réaliser, il fallait au moins que la mer de Champlain se retire et que des irrégularités des reliefs permettent des déplacements de masses. À ce dernier sujet, il est probable que le fond marin en émersion n'était pas uniforme et que les dénivellations topographiques étaient suffisantes pour décider des tassements et des glissements sous l'eau, toute autre chose étant égale (Mary et Dangeard, 1970). Sur le versant laurentien septentrional, au moins trois autres situations pouvaient créer des dénivellations favorables au glissement; l'apport d'eau fluvioglaciaire venant d'un front d'Inlandsis fondant, suivant l'époque, à des distances variables, pour un temps à $160 \mathrm{~km}$ (100 milles) de la haute terrasse ; les courants littoraux de flux et de reflux marins, probablement tout autant puissants qu'irréguliers, comme l'étaient les débits de fonte précédents; I'action strictement fluviale (eau venant surtout des précipitations) apparaît faible par rapport aux deux agents précédents. Dans les vastes dépressions d'encadrement, ces actions se combinaient et s'exerçaient dans le même talweg. Suivant une théorie un peu trop schématique, au fur et à mesure que la mer se retirait, l'encaissement s'accentuait et des terrasses fluviales se dégageaient; les glissements pouvaient alors bénéficier d'un commandement de relief plus hardi ; l'appel de la gravité s'exerçait plus librement. Un cas souvent répété de l'influence fluviale sur les glissements consiste dans l'érosion vive faite par un méandre d'un cours d'eau au pied d'un versant raide.

Du côté anthropique, les causes provocantes sont aussi diverses que l'action des hommes. Tout type de vibrations - barre du son des avions à réaction, tir - et tout type de surcharge en édifices ou en voitures de transport au-delà du seuil de portance peuvent contribuer à la descente brutale de matériaux. Les déboisements et la surculture ont souvent été invoqués pour rendre compte des catastrophes, peut-être trop car les glissements ont commencé avant l'occupation humaine.

Les spécialistes reconnaissent qu'il n'existe pas de cause unique, universelle, qui rendrait compte de toutes les situations. Cette constatation conduit Hurtubise (Canada, 1957) à s'exprimer ainsi : d'un cas récent à l'autre, "les mécanismes sont de nature différente: érosion, structure et pression artésienne à Rimouski : stratification prononcée et infiltration au Lac-Saint-Jean; stabilité décroissante à Nicolet" (page 329). Il faut donc retenir la diversité des conditions qui interviennent dans les glissements.

Mais la littérature a-t-elle fait appel à toutes les causes possibles? S'est-elle assez interrogée sur les conditions des glissements anciens? Le modèle n'est-il pas trop près de la situation présente ? Le fait d'éviter de discuter d'un pergélisol (sans doute discontinu) est-il devenu une preuve 
de son inexistence? Les hypothèses des pays tempérés rendent-elles bien compte des cas aberrants ? A-t-on jamais pensé à considérer côte à côte glissements et autres cavités en creux ? À toutes ces questions, autant de réponses négatives semblent s'imposer. De toute façon, les connaissances courantes ne prouvent pas que l'on peut se passer d'autres hypothèses.

\section{II - GLACE DANS LE SOL ET POSTGLACIAIRE SONT-ILS INCOMPATIBLES? ${ }^{11}$}

Avant de présenter I'hypothèse d'un cycle (ou de cycles) de glace du sol discontinu dans la vallée du Saint-Laurent depuis la déglaciation, il est peut-être utile de rappeler quelques exemples de phénomènes semblables pris dans les régions polaires actuelles. Ces informations comparatives ont leur limite toutefois car il n'est pas du tout prouvé que la Laurentie a alors connu des situations équivalentes à celles que l'on rencontre aujourd'hui dans des secteurs de l'Arctique. Il y a quand même similitude de certaines situations. L'on peut se demander si la démarche utilisée par J.R. Mackay dans le delta du Mackenzie serait totalement déplacée pour les hautes terrasses laurentiennes, il y a environ $9000-8000$ ans? "The most obvious sign pointing to the presence of abundant segregated ice is the ubiquitous slump seen along oversteepened coasts ", écrit-il. Deux phénomènes seront considérés : des glissements périglaciaires et des dépressions thermokarstiques.

D'abord à l'intérieur du Norman (North North America, d'après K. Stone). Des glissements sur place et des "incavations" de thermokarst ont été souvent décrits depuis la construction de la route d'Alaska en 1942. En outre, Lamothe et Saint-Onge (1961) ont montré pour la région d'lsachsen comment la mise à nu et la fonte d'une masse de glace, l'appel de la gravité, la gélifluxion et une certaine fluviation ont déterminé l'évacuation du mollisol local. De son côté, J.R. Mackay écrit en 1966 au sujet du bas delta mackenzien "Ground ice slumps result from the melting of supersaturated frozen grounds, below the active layer (page 68); the slumps resemble the earthflows of more temperate region but the pattern of slumping is quite different "; l'abrupt y recule de 1,5 à 4,5 m (5 à 15 pieds) par an. Dans le Baffin méridional, au nord-est de Lake Harbour, des terrasses pergélisolées sont érodées par des glissements, le tout laissant un paysage bien semblable à celui de certaines cavités laurentiennes. Il en est ainsi du moyen Mackenzie et de la basse vallée de la Rivière aux Liards. Dans le Nord, on trouve évidemment de nombreuses dépressions thermokarstiques (Black, 1969 : Tedrow, 1969). Pour sa part, A. Cailleux a tôt été intégré par la rotondité d'un nombre infini de mares en Amérique du Nord et il met certaines d'entre elles en regard de la glace dans le sol ${ }^{12}$. Le Périglaciaire

11 Cette section constitue l'objet d'une communication au congrès de l'Association Canadienne Française pour I'Avancement des Sciences (ACFAS), Sherbrooke, 1971.

12 CAILLEUX, A., "Observations sur quelques lacs ronds nord-américains", Cahiers de géographie de Québec, no 6, 1959, p. 139-147. Voir aussi Cailleux, 1971. 
par l'image (Hamelin et Cook, 1967, p. 38) donne une photo montrant I'association des mares de thermokarst et des fentes de glace pour l'Arctique canadien central. Cas particuliers qui pourraient être utiles pour une meilleure compréhension des ravins inscrits dans les hautes terrasses du Québec méridional, l'association des rainures d'érosion aux sillons périphériques des polygones de toundra sur les terrasses de la rivière de la Paix en Colombie orientale ${ }^{13}$.

Dans le Norurss (Nord de I'U.R.S.S.), les études de glace dans le sol sont plus avancées qu'en Amérique du Nord; la terminologie aussi. Le moins que l'on puisse rappeler concerne les alass ou dépressions de fusion de la glace du sol ; les auteurs russes (Katasonov, 1969) ont même distingué au moins quatre stades d'évolution :

1. Alass à micro-dépressions isolées et élémentaires ;

2. Alass à dépressions complexes et jumelées ;

3. Vallée d'alass laissant de part et d'autre des buttes témoins appelées inter-alass ;

4. Le post-alass lorsque le niveau encaissant de la phase 1 a disparu : cet âge peut voir apparaître une nouvelle famille de formes froides, par exemple des pingos.

Dans le Québec méridional, si ce processus s'est exercé, le stade 2 n'a pas dû être dépassé. En URSS, la glace dans le sol est de diverses origines; le long de la Léna et de l'Aldan, I'on voit entre autres des fentes de glace. Celle-ci influence la fusion différentielle du sol gelé, tantôt la favorisant (par exemple à Shuyskaya) tantôt en la défavorisant (par exemple à Kuranakh) ; dans le premier cas, un sillon va s'inscrire sous le site d'un coin de glace laissant l'entre sillon en domination topographique ; ces coupoles dont les côtés deviennent convexes s'appellent baidzherakhs et elles sont bien développées à Mamontova Gora sur l'Aldan (photo 6). De tels rappels de la littérature scientifique peuvent aider au dépistage des concepts opératoires dans l'étude du postglaciaire laurentien.

\section{1 - Comment la glace dans le sol aurait-elle pu s'installer?}

Les types de glace dans le sol sont nombreux et ils ont fait l'objet de maintes classifications. "Suivant une typologie tenant aux conditions de congélation, quatre principaux types; engel superficiel des sédiments humides, glace de ségrégation interne, glace développée dans des cavités ouvertes à la surface (fentes), glace profonde d'injection (pingos). L'on pourrait ajouter la glace allogène (glace de glacier; glace flottante) et le glaçage » (Hamelin et Cook, 1967).

13 LAFAY-HAMELIN, C., Le Canada, Montréal, Éd. du Renouveau Pédagogique. 1970 , p. 60 . 


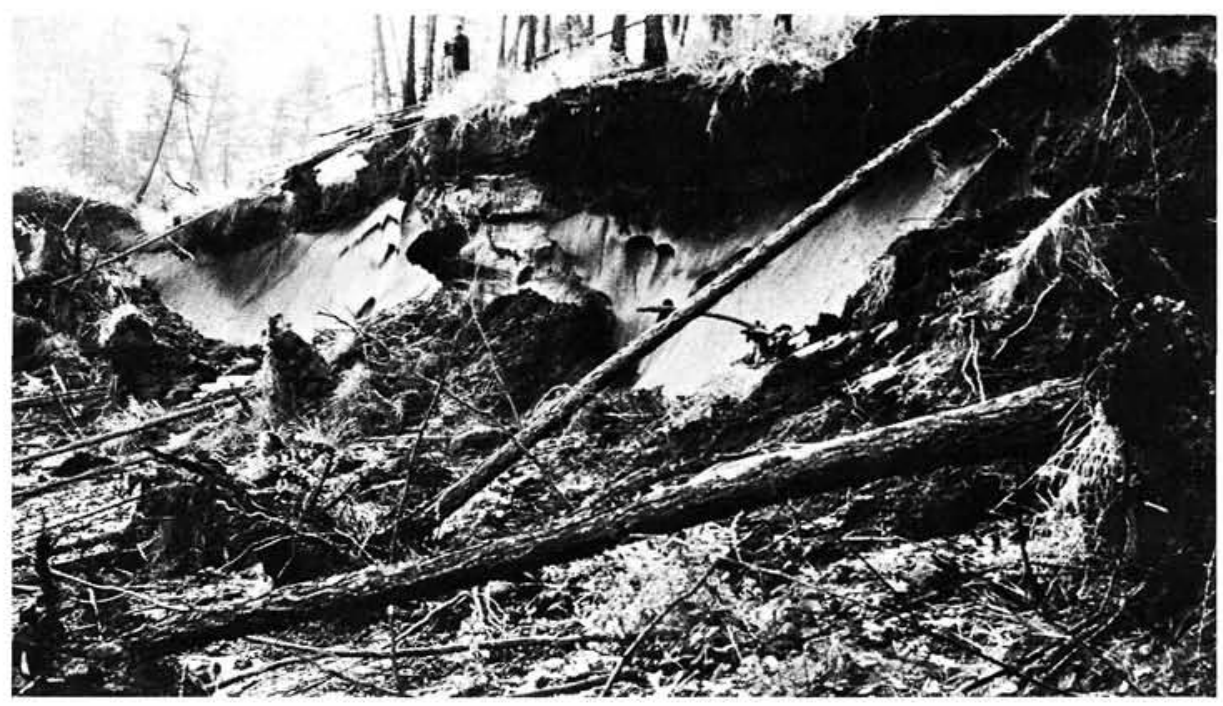

Photo Louis-Edmond HAMELIN (1969)

Photo 5 Glissement sur glace qui entraîne des arbres. Bassin de la Léna, Sibérie nordique.

Dans le haut delta de la Mauricie en particulier, nous croyons que les deux principaux types de glace auraient pu venir de la congélation de l'eau interne circulant en faible profondeur et de l'eau pénétrant dans les fissures ouvertes à la surface. Pour des raisons différentes, avant comme après la mer de Champlain, la glace pouvait être syngénétique ou intraformationnelle

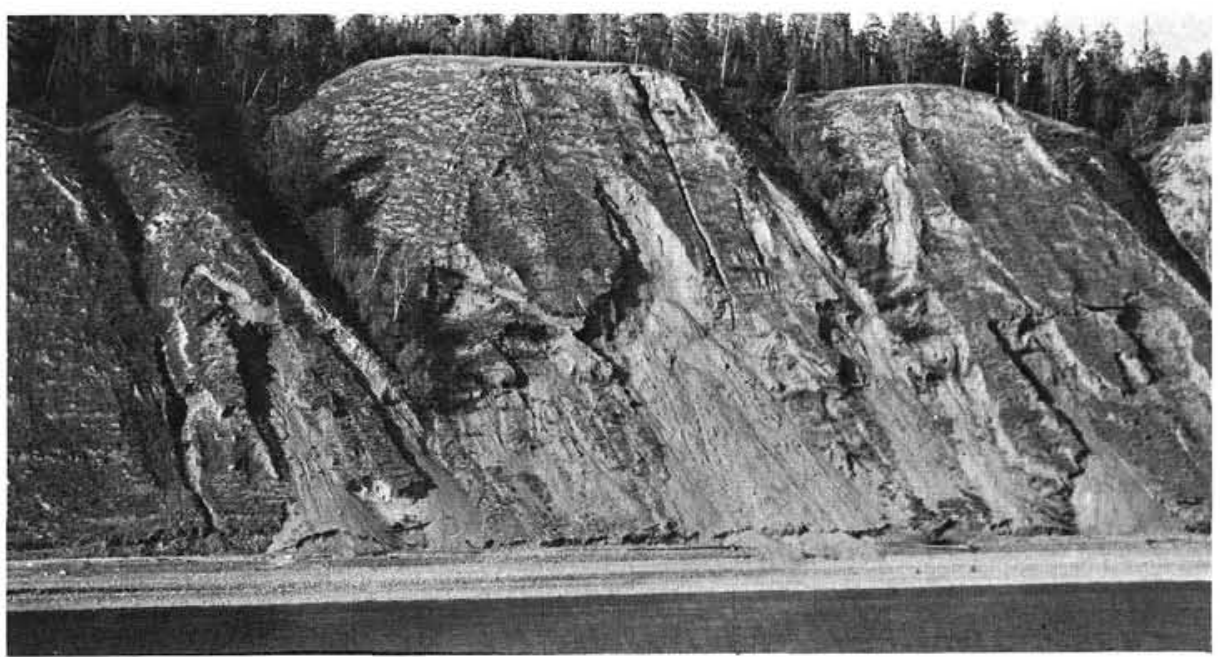

Photo Louis-Edmond HAMELIN (1969)

Photo 6 Sillons installés sur des fentes de glace. Glissements de gravité sur les côtés des baidzherakhs. Mamontova Gora sur I'Aldan, Sibérie nordique. 
(contemporaine de la sédimentation) et épigénétique (postérieur à la sédimentation). La glace interne pseudo horizontale pouvait se présenter en fuseaux lenticulaires ou en champs amorphes.

Mais sur quoi peut-on faire reposer l'hypothèse de l'englacement? Peu avant l'arrivée de la mer de Champlain, la bordure nord déjà déglaciée de la future plaine laurentienne se trouvait située très près d'un front d'inlandsis et, strictement, elle était en position périglaciaire. Peu de temps après le départ de la mer de Champlain et plus tard du golfe de Laflamme (9500 à 8500 B.P.), le front glaciaire avait certes reculé, mais il demeurait dans le bassin laurentien; le périglaciaire en était toujours un de situation. II est plus que probable que des vents froids, katabatiques, descendaient directement du front glaciaire vers la plaine; avec un bord de calotte épais de $300 \mathrm{~m}$ (1000 pieds) seulement, la dénivellation totale

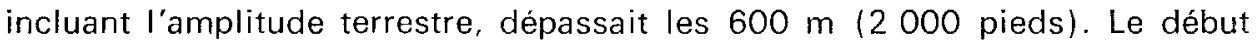
de l'émersion champlainienne a dû être favorable à l'engel de l'eau et des matériaux. Jean-Claude Dionne a fait appel à de semblables conditions proximales pour rendre compte des nombreuses fentes en coin au sud-est de l'estuaire du Saint-Laurent pendant une période un peu antérieure à celle considérée ici. À ce périglaciaire de position, s'est très vraisemblablement ajouté un périglaciaire plus strictement climatique (celui qui ne tient pas d'abord à la proximité du front glaciaire); le climat n'a sûrement pas toujours été comme il l'est aujourd'hui; des périodes plus froides longues de centaines d'années et d'ailleurs répétées à quelques reprises sont choses fort vraisemblables, comme nous le verrons. Par rapport aux données actuelles, la quantité supplémentaire de froidure nécessaire à un englacement discontinu et peu profond n'est pas astronomique: des lentilles pluriannuelles de glace dans le sol n'auraient-elles pas été trouvées en Abitibi ? Des poches de pergélisol persistent au sommet de la Gaspésie et, d'après J. Ives (1962), au sommet des Laurentides.

L'englacement est un fait relatif. De part et d'autre du Champlain, les dépôts minéraux étaient peu protégés de l'air ; la végétation ne faisait que s'installer et il se peut même que l'épaisseur du manteau nival annuel était faible (autrement, il aurait menacé la déglaciation).

Mais l'eau dans le sol était très abondante, surtout en bordure des Laurentides qui véhiculaient de forts débits de fonte; cet apport pouvait fournir l'eau nécessaire à la formation des lentilles épigénétiques et syngénétiques de glace, en dehors des périodes de submersion. Comme on I'a constaté en URSS, il a pu se produire aussi des infiltrations même dans l'argile marine (au travers des fissures, notamment).

Si glace il y a eu, celle-ci formait des îlots discontinus et peu profonds installés dans les dépressions où se concentrait naturellement une partie des eaux. Par ailleurs, le sommet des terrasses exposé au vent et ne possédant pas de tapis nival épais devait contenir des fentes de glace, installées dans les fissures de froid (de gel). L'occupation de la glace était donc différentielle 
et limitée: il ne s'agit pas d'un pergélisol installé à la grandeur de la Laurentie. Cependant, il est vraisemblable de penser qu'au-dessus des masses internes de glace ou qu'à côté des fentes de glace, il y ait eu, comme aujourd'hui, un gélisol saisonnier.

Puisque la glace aurait été discontinue, une certaine circulation d'eau libre et des champs non gelés existaient ici et là. Les occasions de pression locale sur des sédiments — d'ailleurs mal consolidés, redressés inversement à la pente structurelle par le relèvement isostatique - étaient sans doute nombreuses. Á cause de la multiplicité des agents au travail, le modelé final ne pourrait être qualifié de périglaciaire seulement. Puisque ces enrichissements en glace ont disparu — de même qu'une bonne partie des terrains dans lesquels ils s'étaient installés — I'on peut parler d'un cycle de glace dans le sol, ou probablement mieux de cycles. Quant à la fusion, elle comporte aussi des aspects glaciologiques et géomorphologiques.

\section{2 - Modalités et effets possibles du déglacement}

La dispartition d'une glace discontinue et peu profonde peut se faire soit au cours d'une amélioration climatique générale et marquée, soit petit à petit et suivant des conditions saisonnières locales. En Laurentie, les deux ont pu jouer; le premier cas étant quasi nécessaire pour le déglacement différentiel des tourbières; le second a pu réaliser une bonne partie du déglacement des terrasses sablo-argileuses. Si l'englacement est venu par situation à proximité du glacier et le déglacement par des causes avant tout locales, l'amplitude climatique d'un tel cycle de glace dans le sol était donc réduite.

Parmi les conditions non générales qui ont pu favoriser la disparition de la glace dans le sol, il y a l'eau saisonnière de ruissellement; celle-ci peut fournir la chaleur et l'énergie nécessaires à la fusion progressive des fentes de glace, par exemple. Par ailleurs, l'encaissement des cours d'eau pouvait mettre à jour des masses internes de glaces qui, au contact de l'air de l'été, allaient fondre par tranche. Cette eau libérée multipliait les occasions de glissements par bloc ou par flux, partant mettait de nouvelles surfaces gelées au contact des agents de fusion. Ainsi, il n'est pas nécessaire d'avoir une phase climatique nettement plus chaude pour libérer un territoire d'une quantité limitée de glace. "Thermokarst processes usually start as a local increase of the seasonal thawing depth" (Katasonov, 1969, p. 32). Dans I'Arctique, il se produit actuellement des glissements de terrain sur glace sans que I'on ait conscience d'une phase de réchauffement généralisée. "Les conditions du développement du thermokarst ne s'exercent que localement et elles ne sont pas un indice d'un réchauffement climatique général de la région" (Kolasinska, 1970). De toute façon, le déglacement -- si au préalable il y avait eu englacement - ne pourrait étonner vu que des périodes plus chaudes que celles de maintenant se sont produites au cours du Postglaciaire. 
Le déglacement installe un modelé pouvant être qualifié de gélifluidal. La descente des matières, favorisée par une nappe sous-jacente d'eau de fonte, intéressait la tranche supérieure des terrasses; ce genre de glaciosolifluxion ne pouvait se produire que si les autres conditions précédemment décrites étaient favorables, telle une dépression en contrebas pouvant accueillir la masse à déplacer. À ces glissements superficiels ou pelliculaires, s'ajoutent des coulées boueuses, un écoulement fluvial concentré, un écoulement fluidal en glacis, des écroulements de surplomb ou d'abrupts subverticaux, des migrations de blocs gelés dont J. Dylik a décrit minutieusement des paléoformes en Pologne. Les déplacements ne se limitent pas à la surface; il pouvait s'en faire en profondeur suivant des tracés d'écoulement ou des tassements proprement sédimentologiques. Paul Macar parlait de "poches d'effondrement». II va sans dire que les glissements et surtout les coulées boueuses produisent des turbations stratigraphiques. G. Godbout en a relevé lors de ses recherches pédologiques dans Portneuf. J.-C. Dionne, à propos d'un glissement chicoutimien, parle "d'inclusion de sables, de déformation de sédiments argileux, d'apports organiques et de fossilation de blocs glaciels » (1971). Tout est loin de s'être fait d'un seul coup ; au contraire, ces actions se sont répétées chaque fois que l'évolution du relief local redétruisait des équilibres. Il reste donc peu des sites des premières manifestations du déglacement.

Du côté des rainures anciennement installées, il se pourrait que plusieurs d'entre elles aient exploité un sillon (début de la fusion) de fente de glace, phénomène rappelant les rainures d'érosion autour des baidzherakhs. Des ravins subactuels peuvent avoir partiellement emprunté leur tracé à un ancien réseau de fentes; réseau occupé par des dépôts de remplissage au moment de l'inscription de la rainure; en ce cas, ce ne serait pas une légère dépression topographique qui aurait attiré les eaux de ruissellement mais des entonnoirs remplis de sédiments différents.

Par suite des glissements latéraux, des évacuations fluviales longitudinales et des subsidences thermokarstiques, il s'est produit une certaine réduction du relief, et davantage dans les zones de glaces abondantes qu'ailleurs ; celles-ci pourraient contribuer à la répartition différentielle des glissements et des rainures; la fréquence de ces phénomènes ne tiendrait donc pas seulement à des différences sédimentologiques et chronologiques. Par analogie, I'on pourrait considérer certaines dépressions des «pseudoalass". Cette réduction n'a pas complètement détruit la surface originelle et demeurent des buttes témoins, semblables aux interalass et parfois très curieusement situées par rapport au réseau hydrographique actuel ou au réseau antérieur probable. En certains endroits, une mamelonnisation des terrains a pu se réaliser ; sommes-nous en présence de pseudo-baidzherakhs ?

Dans la très grande majorité des cas, l'étendue du territoire occupée par des poches de glace a constitué une superficie plus réduite que celle qui a été influencée par les mécanismes déclenchés par la fusion de cette glace. 
Les cavités anciennes de glissement dont le cadre n'a pas été détruit par la suite sont demeurées figées ou elles ont été partiellement réactivées : les formes d'évolution se limitent à des micro-glissements, à du creep, à des petits entonnoirs, à des surfaces limitées de badlands, à des pieds de vache. En certains cas cependant, les remaniements peuvent atteindre des dimensions importantes, par exemple à Saint-Jean-Vianney, en 1971. À Saint-Joseph-de-Beauce, des cavités de versant sont en train de se transformer en niches nivales. Quant aux rainures inscrites dans les terrasses, elles ont tendance à s'allonger en amont à même la plaine et, en aval, au-delà du talus qui auparavant constituait leur limite propre; dans le fond du vallon, un léger encaissement récent dégage la terrasse dite du Didacien, comme à Gentilly, à Saint-Sévère, à Saint-Cléophas et à Brownsburg. Sur la partie basse des versants et des talus, le principal réaménagement consiste dans un "glacis» à faible pente, suivant l'opinion de R. Raynal.

\section{3 - Limites et mérites de l'hypothèse périglaciaire}

La grande objection que I'on ne manquera pas de dresser contre notre hypothèse viendra des indications climatiques dérivées des études paléobotaniques. Les palynologistes et probablement certains géomorphologues à leur suite diront qu'ils ne trouvent pas de preuves de froid dans les profils végétaux. Cela pourrait clore toute discussion mais peut-on regarder les choses de plus près, à la suite de nombreuses remarques méthodologiques faites à la réunion de I'AMQUA en 1970 dans le Montana ? D'abord des commentaires qui diminuent l'absolu des enseignements suggérés par les pollens. R.A. Bryson rappelle qu'aux changements climatiques, la végétation répond plus rapidement que la glace du sol; dans ce cas, des pollens de la nouvelle période (surtout s'il s'agit de la première) ne prouveraient pas l'inexistence des conditions froides pouvant encore influencer l'évolution des terrains. D'autres auteurs ont signalé que les pollens renseignent moins sur le climat annuel que sur le climat de la saison de croissance; dans le cas des espèces à forte tolérance, la végétation, reflet de la situation de l'été, dit donc peu de chose de l'amplitude saisonnière et des autres conditions. En outre, faut-il rappeler que coexistent glace dans le sol et végétation; nous l'avons signalé pour l'extrémité des langues glaciaires dans le sud-est de l'Alaska : de son côté, la Sibérie centrale montre également qu'une taïga de belle venue croît à quelques pieds seulement au-dessus de lentilles de glace; les deux faits n'expriment donc pas un seul caractère climatique : la glace dans le sol tient à un bilan déficitaire et à long terme alors que la forêt signale les chances pluviothermiques de l'inter-hiver; dans un tel cas, les pollens forestiers recueillis dans les cavités de glissement sur glace de sol ne pourraient prouver l'absence de cette glace. L'évolution d'un relief local peut donc être commandée par la fusion d'une glace interne pendant que les pollens dominants à recueillir exprimeront autre chose. De telles situations invitent à la prudence dans l'utilisation du "climat des botanistes" surtout si le site du piège végétal n'est pas géomorphologiquement représentatif et si les paléochangements ont été surtout caractérisés 
par des éléments autres que ceux de l'été. De toute façon, ce n'est pas n'importe quel profil pollinique qui va prouver l'absence d'une glace discontinue dans le sol. Même, la technique du radio-carbone conduit à des inquiétudes et R.B. Morrisson a signalé au lac de Bonneville des conflits entre la chronologie établie en laboratoire et la stratigraphie relevée sur le terrain. Le moins qu'on puisse dire, c'est qu'il persiste beaucoup d'incertitudes sur la gamme des climats au début du Postglaciaire.

Même s'il s'est fait un réchauffement très général depuis le dernier maximum glaciaire et que les périodes "chaudes " l'ont emporté en intensité et en durée sur les périodes "froides", des refroidissements se sont produits dans les pays de l'Atlantique nord. Suivant une terminologie européenne et une datation étatsunienne, quatre moments: le Dryas récent il y a 10600 ans B.P., le Boréal vers 8000 B.P., le Sud-Boréal autour de 4000 à 2000 B.P. et pendant le présent millénaire. Au cours de ces périodes, surtout les deux premières, un certain englacement aurait pu se produire. Avec la période de "périglaciaire de situation ", il y aurait donc eu plus d'un moment d'empergélisolation possible.

Du côté morphologique aussi, l'hypothèse de la glace dans le sol ne semble pas être improbable. Cet englacement semble être en convergence avec d'autres manifestations du froid, dans les modelés laurentiens. G. Ritchot a déjà souligné l'association des hautes terrasses, des dunes et même des glissements. D'après notre hypothèse, les dunes pourraient témoigner d'une période froide et les glissements de l'eau de fusion de la glace dans le sol ; des variations thermiques et non seulement pluviométriques seraient à la base de cette évolution apparemment contradictoire. Au Mont-Royal, Gilles Ritchot (1967) a identifié des "paléosols de toundra ". Si les observations de $P$. Gangloff sont exactes, des gélistructures installées dans des dépôts champlainiens au niveau des terrasses supérieures feraient appel à un pergélisol discontinu ${ }^{14}$. Jean Poirier a décrit une paléofente de froid aux llles-dela-Madeleine ${ }^{15}$. Jean-Claude Lasserre songeait à un climat froid pour interpréter des talus d'éboulis dans les Laurentides ${ }^{16}$. Nous ne répétons pas les exemples donnés précédemment qui faisaient appel à une glace du sol ; c'était notamment le cas des figurations à la surface des matériaux organiques ; il nous semble acceptable de mettre en relation climatique les réticulations végétales et les glissements d'une même région, par exemple celles et ceux de Shawinigan. Ajoutons que nous avions identifié un glacier rocheux en Gaspésie (Hamelin, 1961, photo p. 194). À voir les cailloux glaciels associés aux terrasses perchées, un froid au moins d'hiver existait avant aujourd'hui. Les très nombreuses fentes en coin soigneusement étudiées par Jean-

14 GANGLOFF, P., "Structures de gélisols reliques dans la région de Montréal », La Revue de géographie de Montréal, vol. XXLV, no 3, 1970, p. 242-253.

15 POIRIER, Jean, "Fente de gel fossile aux îles-de-la-Madeleine ", La Revue de géographie de Montréal, vol. XXLV, no 3, 1970, p. 319-320.

16 LASSERRE, J.C., "Les pentes raides de la vallée de la rivière du Nord ", Cahiers de géographie de Québec, no 19, 1966, p. 73-88. 
Claude Dionne relèvent plus, d'après son auteur, d'un périglaciaire de situation que d'un périglaciaire climatique; elles sont cependant des formes froides postglaciaires, du moins finiglaciaires ${ }^{17}$. II ne devrait donc pas être surprenant de trouver d'autres phénomènes de glace dans le sol dans le relief postglaciaire laurentien.

En ce qui concerne les cavités de glissement et les rainures, le volume total de l'évidement est considérable; dans les régions très ravinées comme bien des secteurs de la Mauricie et du Saguenay, il est difficile de comprendre que les glissements ne se sont pas faits dans le passé à un rythme beaucoup plus élevé que celui de l'époque historique; la fonte d'une glace dans le sol aurait constitué un moyen de rendre compte de la fréquence ancienne de la descente massive de quartiers de terrasse.

Enfin, la glace dans le sol, probablement accompagnée de cycles gélivaux plus agressifs, pourrait aider à la compréhension de phénomènes aberrants comme les " éviers " du canton d'Alfred en Ontario de l'Est, les buttes rondes dans les tourbières, le suréquipement hydrographique ainsi que la localisation surprenante de certaines cavités, vallées et buttes inscrites dans des sections de la plaine du Saint-Laurent.

\section{CONCLUSION}

La portée des réflexions qui précèdent ne doit pas dépasser celle que son auteur veut lui donner. L'hypothèse de la glace du sol n'a pas pour but de remplacer les explications existantes: elle s'offre comme une donnée que l'on suggère d'ajouter aux autres pour rendre compte de phénomènes complexes et sûrement polygénétiques. II s'agit donc de l'addition d'un autre processus dans l'évolution à reconstituer du modèle postglaciaire. La glace n'aurait été qu'un agent, et qu'un agent non universel, de participation. De plus, son action a été limitée dans l'espace, toutes les régions n'ayant pas été également favorables à l'englacement. De plus, elle a été limitée dans le temps; la glace dans le sol n'aurait intéressé que certains épisodes, d'ailleurs assez courts. L'hypothèse n'est pas encore prouvée mais nous espérons qu'elle ne sera plus presque systématiquement ignorée.

\section{BIBLIOGRAPHIE COURTE 18}

AMERICAN QUATERNARY ASSOCIATION (AMQUA). Abstracts, Bozeman, 1970, 168 pages. Compte rendu par L.-E. Hamelin, dans Cahiers de géographie de Québec, no 33, 1970, p. 377-382.

BÉLAND, J., "Nicolet Landslide ", Reprint. Proc. Geol. Ass. of Canada, vol. 8, no 1, 1956 p. 143-156. BLACK, R.-F., "Thaw Depressions and Thaw Lakes, A Review ", Biuletyn Periglacjalny, no 19, 1969. p. 131-150

17 DIONNE, J.-C., «Formes de cryoturbation fossiles dans le sud-est du Québec » Cahiers de géographie de Québec, no 19, 1966, p. 89-100. Voir aussi du même auteur "Nouvelles observations de fentes de gel fossiles sur la côte du Sud du Saint-Laurent ", La Revue de géographie de Montréal, vol. XXIII, no 3, 1969, p. 307-316.

$18 \mathrm{Ne}$ comprend pas les références bibliographiques portées dans le texte. 
BLACK, R.-F., "Climatically Significant Fossil Periglacial Phenomena in North Central United States", Biuletyn Peryglacjalny, Lodz, no 20, 1969, p. 225-238.

BIULETYN PERYGLACJALNY, The Joint Symposium of the Commission on Periglacial Morphology and the Commission on the Evolution of Slopes of the IGU, Lodz, no 18, 1969, 445 pages.

BROWN, R.-J.-E., "Factors Influencing Discontinuous Permafrost in Canada", Dans The Periglacial Environment, edited by T.L. Péwé, Montréal, McGill Press, 1969, p. 11-53.

BRUNSDEN, D., "Ever-Moving Hillsides ", The Geographical Magazine, London, vol. XLIII, no 11, 1971, p. $759-764$.

CAILLEUX, A., "Lacs en ourson, cernes et thermokarst ", Cahiers de géographie de Québec, no 34, 1971. p. $131-136$.

CAILLEUX, A. et TRICART, J., "Un type de solifluxion: les coulées boueuses 》, Revue de géomorphologie dynamique, Strasbourg, T. 1, 1950, p. 4-46, 15 fig.

CANADA, PHOTOTHĖQUE NATIONALE DE L'AIR, Ottawa.

CANADA, NATIONAL RESEARCH COUNCIL, Papers Presented at the fourth International Conference on Soil Mechanics, London, 1957. Ottawa, 1958. Voir surtout les études de W.J. Eden, C.B. Crawford, J.E. Hurtubise, N.R. Gadd et G.G. Meyerhof.

CANADA, COMMISSION GÉOLOGIQUE, Blackburn, Ontario-Québec. Geomorphology-géomorphologie, Ottawa, 1966, Carte $1264 \mathrm{~A}$.

CANADA, NATIONAL RESEARCH COUNCIL, Associate Committee on Soils and Snow Mechanics, Reports, Ottawa.

CHAGNON, J.-Y., "Les coulées d'argile dans la province de Québec", Naturaliste canadien, vol. 95. no 6,1968, p. $1327-1343$.

CLIBBON, P.-B. et BASTIEN, P., Géomorphologie du Québec méridional. Partie 1 - Rive nord du SaintLaurent, Montréal. Centre de psychologie et de pédagogie, 1969 (54 dispositives avec texte explicatif).

CZUDEK, T. et DEMEK, J., "Thermokarst in Siberia and its Influence on the Development of Lowland Relief ", Quaternary Research, N.Y., vol. 1, 1970, p. 103-120.

DIONNE, J.-C.. "Bibliographie du périglaciaire du Québec", La Revue de géographie de Montréal, vol. XXII, no 2, 1968, p. 175-180 (comprenant plusieurs travaux de J.-C. D.).

DIONNE, J.-C., "Contorted Structures in Unconsolidated Quaternary Deposits, Lake Saint-Jean and Saguenay Regions, Québec ", La Revue de géographie de Montréal, vol. XXV, no 1, 1971, p. 5-33, fig.

DUBÉ, J.-C. et HAMELIN, L.-E., Carte préliminaire de phénomènes périglaciaires du Canada, Québec, Institut de géographie, 1960. Présentée au Congrès de I'UGl à Stockolm et à l'ACFAS.

DYLIK, J., "Le thermokarst, phénomène négligé dans les études du Pléistocène ". Annales de Géographie, no 399, Paris, 1964, p. 513-524.

DYLIK, Jeen, "L'érosion thermique actuelle et ses traces figées dans le paysage de la Pologne Centrale ". Bulletin de l'Académie polonaise des Sciences, vol. 19, no 1, 1971, p. 54-61.

GODBOUT, G., Étude pédologique du comté de Maskinongé, Québec, Ministère de I'Agriculture, 1962, 86 pages.

HAMELIN, L.-E., "Les tourbières réticulées du Québec-Labrador subarctique: interprétation morphoclimatique ", Cahiers de géographie de Québec, no 3, 1957, p. 87-106.

HAMELIN, L.-E., "Périglaciaire du Canada. Idées nouvelles et perspectives globales ", Cahiers de géographie de Québec, no 10, 1961, p. 141-203.

HAMELIN, L.-E. et COOK, F.-A., Le périglaciaire par l'image. Illustrated Glossary of Periglacial Phenomena, Québec, Centre d'Études Nordiques et Presses de I'Université Laval, 1967, 237 pages.

HAMELIN, L.-E. et DUMONT, B., "Un terme pour les fonds de vallée dans le sud-est du Canada ", Cahiers de géographie de Québec, no 9, 1960-61, p. 82-84

IVES, J.-D., Iron Mining in Permafrost, Central Labrador-Ungava, Geographical Paper, Ministère des Mines, Ottawa, no 17, 1962, p. 66-77. Voir figure 2.

KATASONOV, E.-M. et SOLOV'EV, P.-A., Guide to Trip around Central Yakutia. Paleogeography and Periglacial Phenomena, Yakutsk. Académie des Sciences, 1969, 87 pages.

KOLASINSKA, J., "Niektore formy krasu termicznego w Centralnej Jakucji (résumé : Certaines formes du thermokarst en Yakoutie Centrale)", Problemy Czwartorzedu, Lodz, 1970, p. 277-284.

LAFLAMME, J.-C., "L'éboulis de St-Alban". Mémoires, Société Royale du Canada, T. XII, Sect. IV, 1895, p. $63-70$.

LASALLE, P., "Late Quaternary Vegetation and Glacial History in the St. Lawrence Lowlands, Canada ", Geologische Medelingen, Leiden, vol. 58, p. 91-128.

LASALLE, P. et CHAGNON, J.-Y., "An Ancient Landslide Along the Saguenay River, Ouébec", Can. J. Earth Sc., vol. 5, no 3, 1968, p. 548-549.

LAMOTHE, Cl, et SAINT-ONGE, D., "A Note on a Periglacial Erosional Process in the Isachsen Area, N.W.T. ", Geographical Bulletin, Ottawa, 16, 1961, p. 104-113.

LAROCHELLE, P., "Soil Mechanics", dans Background Papers on the Earth Sciences in Canada, Geological Survey of Canada, Ottawa, no 69-56, 1970, p. 151-155.

LAVERDIĖRE, C., "La coulée d'argile de Nicolet", Revue canadienne de Géographie, vol. X, no 2-3, 1956, p. 142-147.

LAVERDIĖRE, C., (ouvrage publié sous la direction de) Le Quaternaire du Québec, numéro spécial de La Revue de géographie de Montréal, vol. 23, no 3, 1969, p. 227-391. 
MACKAY, J.-R., "Segregated Epigenetic Ice and Slumps in Permatrost, Mackenzie Delta Area, N.W.T.", Geographical Bulletin, n" 8,1966 , p. 59-80

MACKAY, J.-R., "The origin of Massive ICy Beds in Permafrost, Western Arctic Coast, Canada ", Canada, Canadian Journal of Earth Sciences, vol. 8, ni 4, 1971, p. 397-4.22.

MARKOV, K.-K. et alii., Paleogeography and Pleistocene Phenomena l/pper Volga, Moscow, Académie des Sciences, 1969, 70 pages (en russe et en anglais).

MARY, G, et DANGEARD. L., "Les phénomènes de glissement dans le domaine marin 》, R. de Géographie phy. et de Géol. Dynamique, Paris, vol. XII, no 4, 1970, p. 313-324.

MORIN, LÉO-G., "La coulée d'argile de Saint-Louis (Comté de Richelieu)", dans Naturaliste canadien, 74 (1947), p. 125-143, fig.

POPOV, A.-I., "Underground lce of Northern Eurasia ", Problemy Czwartorzedu, Lodz, 1970, p. 365-369.

POULIOT, A., Le tremblement de terre de 1663 en Nouvelle-France, Dossier manuscrit, Québec, 1963, 68 pages.

PREST, V.-K., "Ouaternary Geology of Canada", from Geology and Economic Minerals of Canada, Ottawa, Ministère de l'Énergie, des Mines et des Ressources, 1970, p. 676-764

QUÉBEC, PHOTOTHEQUE PROVINCIALE DE L'AIR, Québec.

RITCHOT, G., "Le Mont-Royal ". La Revue de géographie de Montréal, vol. XXL, n" 1, 1967, p. $41-79$ et n० 2, p. $267-311$.

ROYAL SOCIETY OF CANADA, Soils in Canada, Spec. Pub!. n 3, 1961, 229 p.

SERGEEV, E.-M., "Les forces de cohésion et l'eau liée dans les argiles". Bull. Bureau Rech. géol. et minier, 2 e série no 1, 1971, section 11, 39, no 399, Paris, p. 9-10 (traduction du russe).

TEDROW, J.-C.-F., "Thaw Lakes, Thaw Sinks and Soils in Northern Alaska", Biuletyn Peryglacjalny, Lodz, no 20, 1969, p. 337-344

TREMBLAY, G., "Conséquences géomorphologiques du relèvement artificiel du niveau du Lac Saint-Jean ", Cahiers de géographie de Québec, no 34,1971 , p.115-130.

TRICART, J., Géomorphologie des régions froides, Paris, P.U.F., 1963, 289 p.

U.S. DEPT. OF COMMERCE, Landslide Investigations, Washington, 1961, $67 \mathrm{p}$.

UNITED STATES, NATIONAL ACADEMY OF SCIENCES, Proceedings, Permafrost international Conference, Washington, 1963,563 pages.

UNION GEOGRAPHIQUE INTERNATIONALE, Commission pour l'étude des Versants. Rapports, Zeitschrift fur Geomorphologie, Gottingen, 1960, 1964. Édité par P. Birot et P. Macar.

ZARUBA, O. et VOLTECH, M., Landslides and their Problems, Prague. Academie des Sciences, 1969, 205 pages, 153 figures.

Depuis la rédaction de notre texte, deux travaux mentionnent des manifestations tardives du froid en bordure des Laurentides.

DIONNE, J.-C., "Fentes de cryoturbation tardiglaciaires dans la région de Québec ", La Revue de géographie de Montréal, vol. XXV, no 3, 1971, p. 245-264.

GANGLOFF, P., CLERMONT, J.-P. et PAQUETTE, G., «Le problème du Tardiglaciaire au Québec méridional ", La Revue de geographie de Montréal, vol. XXV, no 3, 1971, p. 305-308.

Par ailleurs, Daniel LAGAREC vient de nous signaler l'existence de formes thermokarstiques dans la région de Québec.

\section{RÉSUMÉ}

\section{Dans la plaine laurentienne, la glace du sol aurait-elle contribué au façonnement des glissements et autres formes de relief en creux ?}

La formation des glissements de terrain et des rainures sont l'objet de certaines réflexions. Si une cinquantaine de glissements contemporains (depuis 1663) ont fait l'objet d'études, surtout géologiques, environ 700 "coulées" ont été relevées sur photographies aériennes. Les glissements qui correspondent au système "cavité-chaos " sont plus nombreux à la fois sur la rive nord du fleuve et sur les terrasses élevées ; la plupart précèdent la colonisation, donc le déboisement. L'eau constitue la meilleure alliée de l'argile. Les conditions topographiques doivent être également favorables. La gravité joue mais les manifestations tectoniques peuvent favoriser le déclenchement du phénomène. D'autres glissements sont liés à des activités anthropiques. II n'y a pas de cause unique. Les glissements se produisent non seulement dans les "vallées d'encadrement" mais dans les nombreuses rainures ("coulées") érodant les terrasses; la dentritation du tracé des vallons ne doit pas faire conclure à des formes strictement fluviales. L'élargissement des vallées en rapport avec le Didacien comprend trois types. Le modèle des explications usuelles est basé sur des situations présentes mais s'applique-t-il parfaitement aux glissements anciens? 
Nous développons l'hypothèse nouvelle de la glace du sol. Des cas, apparemment semblables, ont été étudiés en Alaska, dans le Nord Canadien et en Sibérie. Dans le sol laurentien, en 1957-58, nous avions déjà signalé des indices de paléo ilôts de glace. Ceux-ci peuvent être de deux types: des masses dans les grandes dépressions, un simple réseau de coins sur des terrasses ou sur des formations fluvioglaciaires; cet englacement a pu s'installer soit pendant un "périglaciaire de situation" au tout début de la déglaciation et de l'émersion, soit pendant des périodes de "périglaciaire climatique" p.e. vers 8000 B.P. ; l'eau était abondante et la végétation mince. Le pergélisol aurait été discontinu. La fusion de cette glace dispersée aurait été liée à des périodes de réchauffement général et à des conditions locales d'exposition à l'air ou à l'eau libre. La fusion aurait favorisé les glissements, les coulées boueuses, les dérangements stratigraphiques et une réduction du relief laissant des buttes témoins. Du côté des rainures, une partie d'entre elles se seraient installées dans des anciennes fentes de froid ou de glace, isolant des quadrilatères, rappelant les baidzherakhs. La glace dans le sol convient très bien aux réticulations végétales qui sont installées sur les hautes terrasses et peut-être aux irrégularités topographiques du canton d'Alfred en Ontario (alass ou thermokarst?). Plusieurs auteurs ont fait appel à des conditions froides pour rendre compte de micro modelés postglaciaires. Le texte discute aussi de l'objection probable des palynologistes. Bref, en Laurentie, la glace du sol aurait été un agent temporaire et non universel qui, depuis la déglaciation, aurait participé à la genèse de certaines formes en creux.

\section{ABSTRACT}

\section{Was ground ice a processus of landscape evolution in the St. Lawrence Lowlands?}

The first part of the text deals with a general review of old and contemporaneous landslides from Ottawa to the Saguenay area. Abundant litterature concerns 'the 1663 earthflow. Near 1840, geologists made study of phenomena of that kind. In sliding, water is the principal allien of clay and silt.

Landslides, ravines, depressions, string bogs, paleo ice wedges and others micro phenomena invite the author to suggest the new hypothesis of discontinuous permafrost in Laurentia during Holocene Time. When the front of Indlandsis is in the Laurentides, coldness was linked with the proximity of glaciers. In other periods, as Boreal Time. one may think of a more like-periglacial climate. Ground ice could be of two types: ice wedges on exposed higher terraces and massive fields in the bottom of wides depressions (Saguenay, Mauricie). The melting of the discontinuous ice could be made according local conditions and general warming. This hypothesis may have facilitate slumping, fluvial dentritation along some ice wedges systems, string vegetation patterns and some thermokarst depressions. The possible objection of palynologists comes into discussions. Ground ice could have been one agent, among others, controlling the evolution of the landscape in some sections of the St. Lawrence Lowlands. 